\title{
An Analytical Model for Lifetime Estimation of Wireless Sensor Networks
}

\author{
Kamanashis Biswas, Student Member, IEEE, Vallipuram Muthukkumarasamy, Member, IEEE, Xin-Wen Wu, \\ Senior Member, IEEE, Kalvinder Singh, Member, IEEE
}

\begin{abstract}
We propose an analytical model to formally define the lifetime of Wireless Sensor Networks by considering various input factors including remaining energy, link quality, and location of the sensor nodes in the network. The model derives an expression for lifetime estimation on the basis of distribution of sensor nodes in the deployment area. The proposed model can be used to evaluate the performance of any routing protocol. We validate the model by means of simulation and show that the analytical outcomes are close to the experimental results.
\end{abstract}

Index Terms-Wireless Sensor Network, Network Lifetime, Link Quality, Retransmission, Remaining Energy

\section{INTRODUCTION}

W IRELESS Sensor Networks (WSNs) consist of a number of resource-constrained sensor nodes (SNs) equipped with sensing, data processing, and radio transmission units. These nodes have finite on-board batteries, which cannot be recharged or replaced in many WSN applications. Therefore, network lifetime is a critical concern in the design of WSN protocols. However, as network lifetime is subject to a variety of input factors, it is a challenge for network designers to accurately model the lifetime of WSNs.

During the past few years, many energy efficient protocols and analytical models have been proposed to extend network lifetime. For instance, the Chen et. al. model derived an expression and identified two key parameters at the physical layer (e.g., the channel state and the residual energy) that affect network lifetime [1]. Based on the formula, a medium access control protocol was also proposed to exploit the parameters. Similarly, sink-centric and energy-based lifetime modeling of sensor networks can be found in [2], [3]. The Sha-Shi model and the Zeng et. al. model consider the relationship between an individual sensor and the entire network, importance of different sensors based on their roles or positions, link quality, and network coverage to define network lifetime. Stochastic Analysis and aVailablity of Energy (SAVE) derived closed form expressions to predict residual energy and lifetime of energy harvesting SNs using stochastic semi-Markov models [4]. Besides these models, extensive research [5], [6] have carried out on lifetime and energy analysis in WSNs.

This paper formally defines network lifetime on the basis of remaining energy of individual sensors, link quality and importance of the SNs in the network. Many applications including large scale WSN use multiple Base Stations (BSs) to ensure reliability and scalability. However, most of the existing network models consider only a single BS located at the center of the network area. These models are, therefore, not applicable for all network scenarios. On the other hand, in the proposed model, multiple BSs can be located anywhere, inside or outside of the deployment area. Furthermore, in contrast of sink-centric topology, the proposed model focuses on the distribution of the SNs over the entire network area. The lifetime of a conventional routing protocol is estimated using both the analytical model and simulation. The results validate the accuracy of the proposed lifetime model.

\section{Modeling Lifetime}

The Lifetime of a Sensor Network (LSN) is defined as the duration (number of rounds) after which the network gets disconnected due to failure of one or more SNs. However, this definition is application-specific. For example, a network can become non-functional either when one $\mathrm{SN}$ or a certain percentage of SNs exhaust their battery power. The basic assumptions of the proposed model are: i) BS and $\mathrm{SNs}$ are stationary, ii) all SNs are homogeneous in terms of their capacity such as communication range and initial energy, iii) every SN is aware of the location of the BS as well as its own, iv) SNs employ multi-hop routing to reach the BS if the BS is out of their communication range. Furthermore, we consider the network area as a collection of unit rectangles where every $\mathrm{SN}$ in a rectangle has equal probability of being selected as a forwarding node. The main benefit of using the grid structure is that the entire network can be divided into sub-networks on the basis of multiple BSs and their area of coverage. Therefore, we can individually measure the lifetime of each sub-network and then combine the results to achieve the total network lifetime. For the proposed model, the remaining lifetime of individual sensor is first derived.

\section{A. Remaining Lifetime of Individual Sensor}

A sensor node consumes energy when it receives, transmits or listens to the wireless channel. For the sake of simplicity, we only consider the energy dissipation involved in sending, receiving and retransmitting messages. The Remaining Lifetime of Individual Sensor (RLIS) can be defined as the ratio of the remaining energy to the initial energy. Thus, Remaining lifetime $=($ Initial energy - Consumed energy $) /$ Initial energy. It can be expressed as shown for the Sha-Shi model [2]:

$$
L(s)=1-\sum_{i=1}^{N_{m}} \frac{\epsilon_{s i q} N_{s i q} R_{t}+\epsilon_{s i r} N_{s i r} R_{t}}{E_{s}}
$$

where, $L(s)$ is the remaining lifetime of sensor node $s ; N_{m}$ is the total number of queries or events; $\epsilon_{s i q}$ and $\epsilon_{s i r}$ are the amount of energy required to transmit and receive a query, and a reply message respectively; $N_{s i q}$ and $N_{s i r}$ are the 


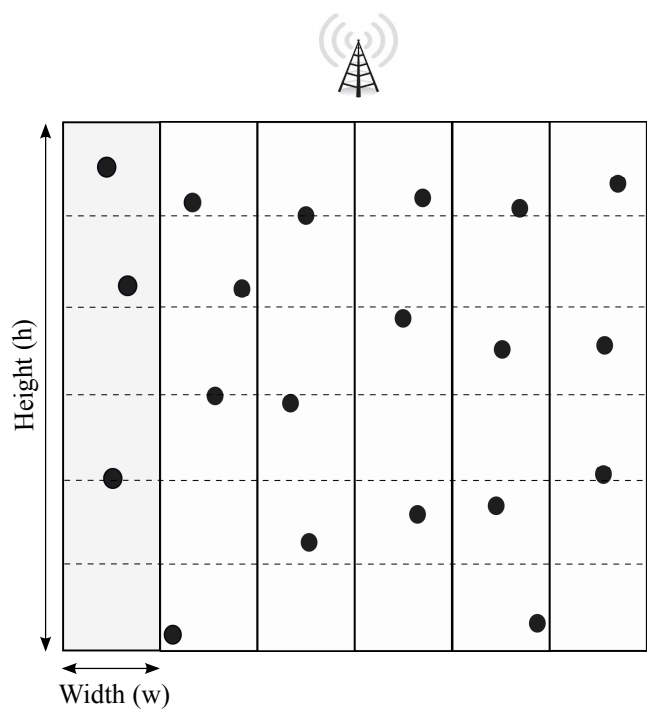

Fig. 1: Deployment area divided into unit rectangles

number of transmitted and received query, and reply messages respectively by sensor $s$ at moment $i$ based on probability $P_{s i} ; R_{t}$ is the expected number of retransmissions under the assumption that query and reply messages experience the same link quality, and $E_{s}$ is initial energy of node $s$. Now, based on the energy model described in [7], we derive the radio hardware energy consumption for transmitting $k$-bit message over distance $d$ (i.e., $E_{T x}$ ) and receiving $k$-bit message (i.e., $\left.E_{R x}\right)$ as follows:

$$
\begin{aligned}
& E_{T x}(k, d)=k \epsilon_{\text {elec }}+k \epsilon_{\text {amp }} d^{2} \\
& E_{R x}(k)=k \epsilon_{\text {elec }}
\end{aligned}
$$

where, $\epsilon_{\text {elec }}$ and $\epsilon_{a m p}$ denote electronics energy to power radio circuitry and amplifier energy for radio signal propagation respectively for one bit information. Thus, the energy consumption can be expressed as: $\epsilon_{s i q}=\epsilon_{s i q . E_{T x}}+\epsilon_{s i q . E_{R x}}$ and $\epsilon_{s i r}=\epsilon_{s i r . E_{T x}}+\epsilon_{s i r . E_{R x}}$; where, $\epsilon_{s i q . E_{T x}}$ and $\epsilon_{s i r . E_{T x}}$ represent energy consumption for sending query and reply messages respectively, $\epsilon_{s i q . E_{R x}}$ and $\epsilon_{s i r . E_{R x}}$ denote energy consumption for receiving query and reply messages respectively.

The probability of a message passing through sensor $s$ at moment $i\left(P_{s i}\right)$ is now calculated in order to compute $N_{s i q}$ and $N_{s i r}$. Since the proposed model is based on the distribution of the SNs over the entire network, the deployment area is divided into a number of unit rectangles both horizontally and vertically as shown in Fig. 1. The basic assumption is that the messages will pass through either vertical or horizontal unit rectangles depending on the locations of SNs and the BS. As an example, if the locations of the BS and a $\mathrm{SN}$ are $(0,0)$ and $(0,70)$ respectively, then the messages have to traverse through horizontal unit rectangles to reach the BS. On the other hand, for a SN with $(70,0)$ location, the messages will go across vertical unit rectangles. Furthermore, since all SNs in unit rectangle have equal probability, the probability of passing a message through a $\mathrm{SN}$ within a rectangle is $\frac{1}{v}$, if there are $v$ sensors in that rectangle. Let $A$ and $B$ be two events, where $A$ denotes the occurrence of a message going through sensor $s$ at moment $i$, and $B$ denotes the event that the destination node is far away from the node $s$ at moment $i$. Since these two events are independent, the probability of a message going through the sensor $s$ at $i$-th moment can be defined as follows:

$$
P_{s i}=P(A)_{s i} P(B)_{s i}=\frac{N-h \rho t}{N h \rho}
$$

where, $P(A)_{s i}=\frac{1}{h \rho}$ and $P(B)_{s i}=\frac{N-h \rho t}{N}$. The term $N$ denotes the total number of SNs, $h$ is the height of the rectangle, $\rho$ is the density of the SNs in the network and $t$ is the number of unit rectangles between the sensor and the BS.

Finally, we calculate the packet retransmission rate in Automatic Repeat reQuest (ARQ) protocol. Let $P_{e}$ be the average packet error rate for the wireless channel, and $G$ be the maximum retransmission attempts allowed by the ARQ protocol. The packet error rate can be expressed as follows: $P_{e}=1-\left(1-P_{b}\right)^{m}$; where $P_{b}$ is the bit error rate of the wireless channel and $m$ is the number of bits in a packet [4]. If a packet is not successfully transmitted after $G$ attempts, then it is dropped. Hence, the expected number of retransmissions for a single packet can be defined as shown below:

$$
\begin{array}{r}
R_{t}=\sum_{k=1}^{G} k F_{t}(k)=\sum_{k=1}^{G-1}\left[k P_{e}^{k-1}\left(1-P_{e}\right)\right]+G P_{e}^{G}=\frac{1-P_{e}^{G}}{1-P_{e}} \\
\text { where, } F_{t}(k)= \begin{cases}P_{e}^{k-1}\left(1-P_{e}\right) & 1 \leq k<G, \\
P_{e}^{k} & k=G\end{cases}
\end{array}
$$

By substituting the above values into Eq. (1), we can express the RLIS as follows:

$$
L(s)=1-\sum_{i=1}^{N_{m}} \frac{\frac{\left(1-P_{e}^{G}\right)\left(\epsilon_{s i q} N_{s i q}+\epsilon_{s i r} N_{s i r}\right)}{1-P_{e}}}{E_{s}}
$$

\section{B. Remaining Lifetime of Sensor Network}

The Remaining Lifetime of a Sensor Network (RLSN) can be obtained by aggregating the remaining lifetime of all individual sensors. However, the location of a SN in the network also has a significant impact on determining network lifetime. For example, the SNs closer to the BS will deplete their energy quickly since they will forward more data packets than the SNs located far away from the BS. This means that the sensors closer to the BS should be given more weights than the distant nodes. Therefore, the distance between the BS and a SN $\left(d_{s b}\right)$ is inversely proportional to the weight factor $\left(w_{s}\right)$ of that node. Thus, $w_{s}=\frac{c}{d_{s b}}$, where $c$ is a constant. Now, by combining the weight factor with remaining lifetime of individual sensors, we can express the RLSN as follows:

$$
\Re=\sum_{s=1}^{N} \frac{c}{d_{s b}} L(s)
$$

\section{Lifetime Estimation FOR CONVENTIONAL PROTOCOL}

In this section, the proposed model is used to determine the lifetime of a network under a conventional protocol such as request and response protocol. In request and response protocol, the SNs send request messages including their current node status (e.g., energy level, number of hops) to the neighbour 
nodes and the neighbour nodes select the best forwarding node on the basis of the received information. The query (request) message is sent to all SNs while the reply message (response) goes through only the sensors selected to establish the communication link. Using Eq. (4) and (6), we can define the RLIS for each query as follows:

$$
\begin{gathered}
L(s)=1-\sum_{i=1}^{N_{m}} \frac{\frac{\epsilon_{s i q} N_{n g h}\left(1-P_{e}^{G}\right)}{1-P_{e}}+\frac{N \epsilon_{s i r} P_{s i}\left(1-P_{e}^{G}\right)}{f\left(1-P_{e}\right)}}{E_{s}} \\
=1-\frac{N_{m} \epsilon_{s i q} N_{n g h}\left(1-P_{e}^{G}\right)}{E_{0}\left(1-P_{e}\right)}-\frac{N_{m} \epsilon_{s i r}\left(1-P_{e}^{G}\right)(N-h t \rho)}{E_{0} h \rho f\left(1-P_{e}\right)}(8)
\end{gathered}
$$

where, $N_{n g h}$ is the number of neighbours of node $s, E_{0}$ and $\frac{1}{f}$ are initial energy and the probability of generating a response by a SN respectively. Now using Eq. (7) and (8), we can calculate the RLSN for conventional protocol as follows:

$$
\begin{gathered}
\Re=\sum_{s=1}^{N} \frac{c}{d_{s b}}\left(1-\frac{N_{m} \epsilon_{s i q} N_{n g h}\left(1-P_{e}^{G}\right)}{E_{0}\left(1-P_{e}\right)}\right. \\
\left.-\frac{N_{m} \epsilon_{s i r}\left(1-P_{e}^{G}\right)(N-h t \rho)}{E_{0} h \rho f\left(1-P_{e}\right)}\right) \\
\approx c \ln \left(d_{\max }+1\right)\left(1-\frac{N_{m} \epsilon_{s i q} N_{n g h}\left(1-P_{e}^{G}\right)}{E_{0}\left(1-P_{e}\right)}\right. \\
\left.-\frac{N_{m} \epsilon_{s i r}\left(1-P_{e}^{G}\right)(N-h t \rho)}{E_{0} h \rho f\left(1-P_{e}\right)}\right)
\end{gathered}
$$

where, $d_{\max }$ denotes the maximum distance between the BS and a $\mathrm{SN}$.

\section{Verification And Analysis of the Model}

The proposed model is evaluated both analytically and by simulation in order to validate its accuracy. For analytical investigation of RLIS and RLSN, we consider the following parameters. The initial energy of each $\mathrm{SN}$ is set to $1.725 \mathrm{~J}$. The energy consumption for sending and receiving a query message is $240 \mu \mathrm{J}$ and $120 \mu \mathrm{J}$ respectively, whereas the energy for transmitting and receiving a reply message is $1200 \mu \mathrm{J}$ and $600 \mu \mathrm{J}$ respectively [2]. The probability of packet error is set to 0.1 , the maximum number of retransmissions is set to 3 , and $c$ is 0.01 . We assume the total of 100 nodes are randomly deployed in a $100 \times 100\left(\mathrm{~m}^{2}\right)$. Thus, the density of the SNs is 1 per $100 \mathrm{~m}^{2}$. Furthermore, we assume the average number of neighbour nodes for an individual sensor is 5 and the probability of generating a response by a sensor is $\frac{1}{30}$, i.e., $f=$ 30. Now, using Eq. (8), we compute the remaining lifetime of different sensors located at different unit rectangles as shown in Table I. From the results, it can be seen that the remaining lifetime of the sensors is increased when the distance between sensors and the BS is increased. This is because the SNs close to the BS consume more energy as they transmit and receive more data packets than the distant SNs.

The remaining lifetime of the network is calculated using Eq. (9). Table II shows the RLSN values obtained for both single and multiple BSs. It can be seen that the RLSN is extended when the BS is located at the center (in the case of a
TABLE I: Remaining lifetime of SNs at different locations

\begin{tabular}{lc}
\hline Unit Rectangles (t) & RLIS \\
\hline 10 & $1-\frac{4316 N_{m}}{10^{6}}$ \\
40 & $1-\frac{3263 N_{m}}{10^{6}}$ \\
70 & $1-\frac{2210 N_{m}}{10^{6}}$ \\
100 & $1-\frac{1158 N_{m}}{10^{6}}$ \\
\hline
\end{tabular}

single BS scenario). As an example, if we set $N_{m}=250$, the amount of remaining energy is 0.035 and 0.012 for location of BS at $(0,0)$ and $(50,50)$ respectively. This remaining energy is completely unused since at that time most of the nodes around the sink are out of power according to Table I. However, the values indicate that the amount of unused energy is low when the BS is located at the center. On the other hand, the RLSN is increased significantly in multiple BSs scenario. In this scenario, the network becomes non-functional when the RLSN value is 0.019 for $N_{m}=780$. Since the packets travel a relatively shorter distance, the networks sustains for more rounds compared to the single BS scenario.

TABLE II: Remaining lifetime of the sensor network

\begin{tabular}{llc}
\hline Number of BS & Location of BS & RLSN \\
\hline Single BS & {$[0,0]$} & $\frac{49487-57 N_{m}}{10^{6}}$ \\
& {$[50,50]$} & $\frac{42626-124 N_{m}}{10^{6}}$ \\
\hline Multiple BS & {$[25,25],[100,0]$} & $\frac{71279-67 N_{m}}{10^{6}}$ \\
\hline
\end{tabular}

\section{A. Simulation Results}

We implement the proposed model in MATLAB to verify the results of the analytical model. For each scenario, 100 nodes are randomly deployed in a $100 \times 100\left(\mathrm{~m}^{2}\right)$ area and simulations are run for 1000 trials to obtain the average performance measures. Table III shows the simulation parameters, which are commonly used in WSN experiments [7].

TABLE III: Simulation parameters

\begin{tabular}{lll}
\hline Types & Parameters & Value \\
\hline Network & Initial Energy & $1.725 \mathrm{~J}$ \\
\hline \multirow{2}{*}{ Application } & $\begin{array}{l}\text { Transmission Range } \\
\text { Packet Size }\end{array}$ & $10 \mathrm{~m}$ \\
& 30,150 bytes \\
\hline \multirow{2}{*}{ Radio Model } & $\epsilon_{\text {elec }}$ & $50 \mathrm{~nJ} / \mathrm{bit}$ \\
& $\epsilon_{\text {amp }}$ & $10 \mathrm{pJ} / \mathrm{bit} / \mathrm{m}^{2}$ \\
\hline
\end{tabular}

First, we calculate the remaining lifetime of the SNs located at different rectangles after running the simulations for 700 rounds. Fig. 2 shows that the nodes located near the BS possess very low energy compared to the nodes at distant locations. The remaining lifetime of the SNs increases with the increase of the distance (unit rectangles). However, the increase in remaining lifetime is not linear for all SNs at 


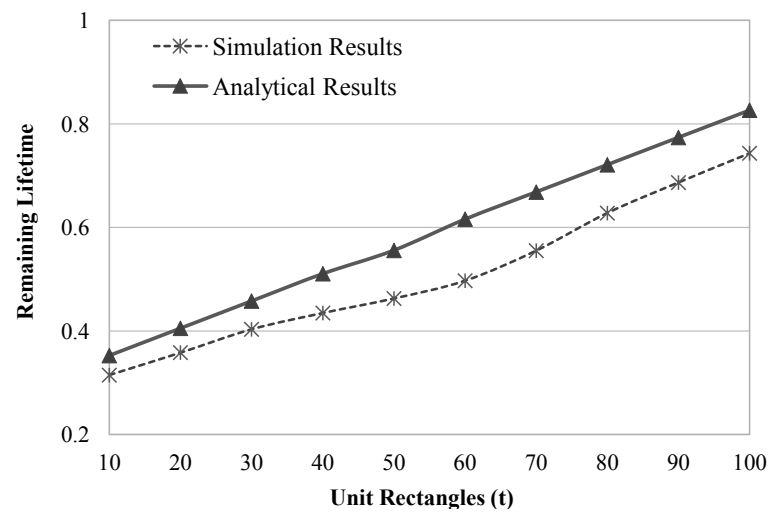

Fig. 2: Remaining lifetime at different locations

different locations. The reason is that the SNs near the center of the network area consume more energy compared to the SNs close to boundary due to increase in network connectivity and collisions. The analytical results also indicate that the closer the $\mathrm{SN}$ is to the BS, the lower the energy it would have. Thus, the simulation results confirm the validity of the analytical model.

Fig. 3 illustrates the simulation results of the remaining energy of the network. From the figure, it can be seen that the remaining energy of BS at $(0,0)$ location is lower than that of BS at $(50,50)$ until three nodes run out of energy. Since packets travel a long distance to reach the BS, a significant amount of energy is consumed in receiving and forwarding data packets. However, after that the remaining energy for BS at $(0,0)$ location increases and the trend is continued for rest of the dead nodes. When more nodes exhaust their battery, the network is partitioned and many SNs cannot communicate with the BS in spite of having a sufficient amount of energy. In contrast, SNs remain alive for more rounds and consume more energy if the BS is positioned at the center. Therefore, the topology makes better use of the node energy compared to the $(0,0)$ location in the long run. Both simulation and analytical results (Table II) show that deploying the BS at the center significantly extends network lifetime.

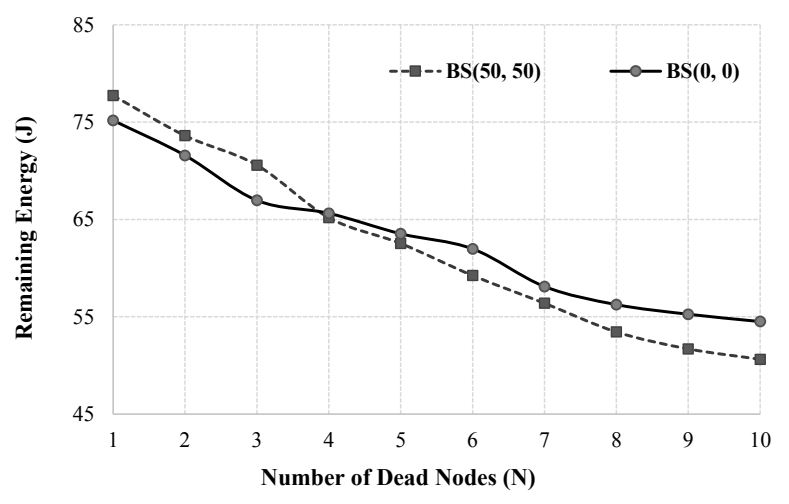

Fig. 3: Remaining energy of the sensor network

Fig. 4 shows the total number of rounds until the network becomes non-functional. The network is considered nonfunctional when $10 \%$ of the total nodes is dead. From the graph, it can be seen that the network sustains for 868 rounds

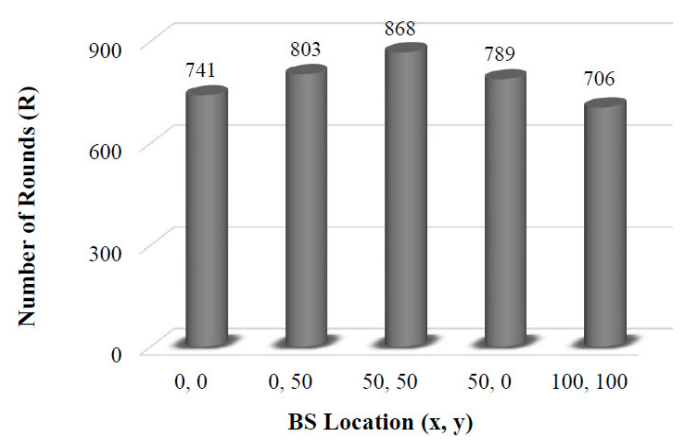

Fig. 4: Network lifetime for different BS locations

for the center location whereas it remains alive for 741 and 706 rounds for the corner positions. The BS located at the corner or edge is surrounded by fewer SNs compared to the BS at the center. Thus, the network goes down when the surrounding nodes run out of power. This leads to the network partition and waste of a significant amount of energy. However, both the remaining energy and network lifetime analysis validate the results shown in Table II.

\section{CONClusion}

This letter presents an analytical model to define the network lifetime of WSNs. Unlike the sink-centric approaches, the proposed model focuses on the SNs distributed over the entire network. Moreover, the model provides the flexibility of using multiple BSs as well as positioning the BSs anywhere, inside or outside of the network area. The experimental results also show the accuracy of the proposed analytical model. For future work, we aim to integrate other energy consumption activities such as state transition, listening to the communication channel and data processing to the model.

\section{REFERENCES}

[1] Y. Chen, and Q. Zhao, "On the Lifetime of Wireless Sensor Networks," in IEEE Communication Letters, vol. 9, no. 11, pp. 976-978, 2005.

[2] K. Sha, and W. Shi, "Modeling the Lifetime of Wireless Sensor Networks," in Sensor Letters, vol. 3, no. 2, pp. 126-135, 2005.

[3] P. Zeng, C. Zang and H. Yu, "Bounding the Lifetime of Target Tracking Sensor Networks," in Proc. IEEE ICC, vol. 8, pp. 3444-3449, 2006.

[4] M. Y. Naderi, S. Basagni, and K. R. Chowdhury, "Modeling the Residual Energy and Lifetime of Energy Harvesting Sensor Nodes," in Proc. IEEE Globecom, pp. 3418-3424, 2012.

[5] D. Jung, T. Teixeira, and A. Savvides, "Sensor node lifetime analysis: Models and tools," in ACM Trans. on Sensor Networks, vol. 5, no. 1, pp. 1-33, February 2009.

[6] F. Kerasiotis, A. Prayati, C. Antonopoulos, C. Koulamas, and G. Papadopoulos, "Battery lifetime prediction model for a WSN platform," in Proc. Int. Conf. on Sensor Tech. Appl., pp. 525-530, 2010.

[7] W. B. Heinzelman, A. P. Chandraprakasan and H. Balakrishnan, "Application specific protocol architecture for wireless microsensor networks", in IEEE Trans. on Wireless Networking, vol. 1, no. 4, pp. 660-670, 2002. 